\title{
Photodynamic Therapy Plus Chemotherapy Compared with Photodynamic Therapy Alone in Hilar Nonresectable Cholangiocarcinoma
}

\author{
Robert Wentrup*, Nicola Winkelmann ${ }^{\dagger}$, Andrey Mitroshkin ${ }^{\dagger}$, Matthias Prager $^{\dagger}$, Winfried Voderholzer ${ }^{\ddagger}$, Guido Schachschal $^{\S}$, \\ Christian Jürgensen ${ }^{\dagger}$, and Carsten Büning ${ }^{*}{ }^{\dagger}$ \\ *Department of Internal Medicine, Waldfriede Hospital, Berlin, ${ }^{\dagger}$ Department of Hepatology and Gastroenterology, Charité-Universitätsmedizin \\ Berlin, Berlin, ${ }^{\neq}$Gastroenterology Practice, Berlin, and ${ }^{s}$ Department of Interdisciplinary Endoscopy, University Hospital Hamburg-Eppendorf, \\ Hamburg, Germany
}

Background/Aims: Standard treatments are not available for hilar nonresectable cholangiocarcinoma (NCC). It is unknown whether combination therapy of photodynamic therapy (PDT) plus systemic chemotherapy is superior to PDT alone. Methods: We retrospectively reviewed 68 patients with hilar NCC treated with either PDT plus chemotherapy (PTD-C) or PDT monotherapy (PDT-M). The primary endpoint was the mean overall survival rate. Secondary endpoints included the 1-year survival rate, risk of cholangitic complications, and outcomes, which were evaluated according to the chemotherapy protocol. Results: More than $90 \%$ of the study population had advanced hilar NCC Bismuth type III or IV. In the PDT-M group ( $n=35)$, the mean survival time was 374 days compared with 520 days in the PDT-C group $(n=33$, $p=0.021$ ). The 1-year survival rate was significantly higher in the PDT-C group compared with the PDT-M group $(88 \%$ vs $58 \%, p=0.001$ ) with a significant reduction of mortality (hazard ratio, 0.20; 95\% confidence interval, 0.07 to 0.58 ; $\mathrm{p}=0.003$ ). Gemcitabine monotherapy resulted in a shorter survival time compared with the gemcitabine combination therapy (mean, 395 days vs 566 days; $p=0.09$ ). Cholangitic complications were observed at a similar frequency in the PDT-C and PDT-M groups. Conclusions: Combining repeated PDT with a gemcitabine-based combination therapy might offer a significant survival benefit in patients with hilar NCC. (Gut Liver 2016;10:470-475)

Key Words: Nonresectable cholangiocarcinoma; Photochemotherapy; Chemotherapy; Survival

\section{INTRODUCTION}

Cholangiocarcinoma (CC) is a malignant neoplasia originating from bile duct epithelia. Although the incidence rate is low $(1-2 / 100,000)$, CC is the second most common primary malignant tumor of the liver after hepatocellular carcinoma. ${ }^{1}$ In total, $5 \%$ to $10 \%$ are intrahepatic, $60 \%$ to $70 \%$ perihilar, and $20 \%$ to 30\% extrahepatic. ${ }^{2}$ The Bismuth classification distinguishes four different categories of hilar CC with respect to localization in the main branches: Type I tumors are limited to the common bile duct with more than $2 \mathrm{~cm}$ from the confluence, type II tumors involve the confluence. Type III tumors involve either right (IIIa) or left (IIIb) hepatic duct while type IV tumors extend to both ducts or are located multifocally. In "Klatskin" tumors, hepatic duct bifurcation is involved. ${ }^{1}$ Hilar CC is often diagnosed at advanced tumor stages making the only curative approach-RO resection-rather difficult.

In hilar nonresectable cholangiocarcinomas (NCCs), different palliative strategies are applied. Apart from best supportive care, adequate and continuous bile duct drainage via endoscopic stenting is the mainstay of palliative treatment. Photodynamic therapy (PDT) is a well-known local treatment approach for NCC.

In PDT, a nontoxic photosensitizing agent such photofrin is given intravenously 48 hours before transpapillary or percutaneous radiation with light of a specific wave length. Due to the accumulation in the neoplastic tissue, the photosensitizing agent generates reactive oxygen radicals after absorption of light resulting in the destruction of tumor cells. In the pioneer study, Ortner et al. ${ }^{3}$ reported a prolonged survival in patients who were treated with stenting with subsequent PDT versus stenting alone.

Systemic chemotherapy is another option for hilar NCC.

Correspondence to: Carsten Büning

Department of Internal Medicine, Waldfriede Hospital, Argentinische Alle 40, Berlin 14163, Germany

Tel: +49-30-818108242, Fax: +49-30-8181077243, E-mail: c.buening@waldfriede.de

Received on March 2, 2015. Revised on July 6, 2015. Accepted on July 23, 2015. Published online January 28, 2016 pISSN 1976-2283 eISSN 2005-1212 http://dx.doi.org/10.5009/gnl15175

@ This is an Open Access article distributed under the terms of the Creative Commons Attribution Non-Commercial License (http://creativecommons.org/licenses/by-nc/4.0) which permits unrestricted non-commercial use, distribution, and reproduction in any medium, provided the original work is properly cited. 
Bile duct tumors are only moderately chemotherapy-sensitive and currently no standard chemotherapy protocols exist. Several different cytotoxic agents have been evaluated, but the results were largely disappointing. Most of the regimens were gemcitabine-based, either as single agent or in combination with 5-fluoruracil (FU) and other agents. Studies evaluating gemcitabine as a monotherapeutic agent or in combination with other agents such as cisplatin, oxaliplatin, docetaxel, mitomycin C, and 5-FU/leucovorin reported response rates up to $60 \% .{ }^{4,5}$ A recent randomized phase 3 study showed that cisplatin plus gemcitabine versus gemcitabine alone was associated with a significant survival advantage in patients with advanced biliary cancer. However, only a subgroup of the patients had hilar CC. ${ }^{6}$

Although local and systemic approaches in hilar NCC are available, trials using a combined approach of both PDT and chemotherapy are limited. We therefore performed a retrospective analysis of 68 patients with hilar NCC that were treated at our centre between 1996 and 2012 with either PDT alone ( $n=35)$ or PDT plus chemotherapy (PDT-C) $(n=33)$. We analyzed numerous parameters such as overall survival including subgroup analysis of the different chemotherapy protocols used, side effects of chemotherapy, and incidence of cholangiocarcinomaassociated complications such as cholangitis.

\section{MATERIALS AND METHODS}

\section{Study protocol}

At first, we retrospectively collected data between 1996 and 2012 on patients in our institution that fulfilled the following criteria: hilar NCC (after interdisciplinary tumor board decision or surgery declined by the patient), treatment with PDT and chemotherapy, and follow up available $(n=33)$. Second, we chose a group of hilar NCC patients treated at the Department of Hepatology and Gastroenterology, Charite, Berlin, Germany with PDT monotherapy (PDT-M) and matched according to the following criteria: age, gender, and Bismuth classification at study entry $(n=35)$. We extracted these patients out of a database of 145 patients that were treated with PDT between 1996 and 2012. A subgroup of this cohort has been published in a previous trial. ${ }^{3}$ None of the patients received an additional radiation therapy.

\section{Data collection}

In total, 68 patients were enrolled in this study. None of the patients had received either chemotherapy or PDT for CC at study entry. The following data were obtained: gender, age, time of diagnosis, Bismuth classification, locally advanced or metastatic disease at inclusion, histology/cytology results, imaging results (endoscopic retrograde cholangiopancreatography [ERCP], ultrasound, computed tomography [CT], magnetic resonance imaging [MRI]), number of PDT sessions, chemotherapy regimen, episodes of cholangitis (defined as fever and/or shaking chills, laboratory evidence of an inflammatory response with abnormal white blood cell count and/or increased serum C-reactive protein, and an increase in liver chemistries, e.g., elevated bilirubin, alkaline phosphatase, $\gamma$-glutamyl transpeptidase, alanine aminotransferase, aspartate aminotransferase), number of hospital admissions, time of death or time of latest followup. Time of death was available in 64 patients. In all patients follow-up data were available for a mean of 18.2 months.

\section{Diagnosis of CC}

Diagnosis of CC was confirmed histologically or cytologically in the majority of cases $(n=50)$. In the remaining cases, diagnosis was based on data from abdominal contrast-enhanced ultrasound, CT scan, MRI, or ERCP, and supported by growth of the tumor lesion during the follow-up.

\section{Endoscopic stenting}

All patients received endoscopic stenting in order to prevent cholestasis. Stent exchange was performed on a regularly basis every 3 months or earlier if signs of stent dysfunction or complications such as cholangitis occurred.

\section{Photodynamic therapy}

PDT was performed according to the protocol as described above. $^{3}$ In brief, patients received photofrin (Photofrin $\mathrm{II}^{\circledR}$; Axcan Pharma Inc., Mount-Saint-Hilaire, Canada) at $2 \mathrm{mg} / \mathrm{kg}$ body weight intravenously 48 hours prior to laser activation. During PDT, endoprostheses were removed at first and then a Cotton-Huibregtse ${ }^{\circledR}$ set catheter (Cook Medical, Bloomington, IN, USA) was introduced proximal to the malignant strictures. Intraluminal photoactivation was performed with a laser quartz fiber bearing a cylindrical diffuser tip (Rare Earth Medical, West Yarmouth, MA, USA; length, $40 \mathrm{~mm}$; core diameter, $400 \mu \mathrm{m}$ ) and an X-ray marker on both sides of the diffuser, which was placed at the proximal end of the catheter. The catheter was then pulled back while the fiber was held in place. Photoactivation was performed at $630 \mathrm{~nm}$ using a light dose of $180 \mathrm{~J} / \mathrm{cm}^{2}$, energy fluence of $0.241 \mathrm{~W} / \mathrm{cm}^{2}$, and irradiation time of 750 seconds (diode laser Visulas 630; Zeiss, Jena, Germany). All patients received oxygen $(4 \mathrm{~L} / \mathrm{min})$ via a nasal catheter to enhance the PDT effect. If tumor length exceeded the maximal diffuser length, an overlap of the fields treated was avoided by a stepwise pull-back of the fiber under X-ray control. In Bismuth IV strictures, a guidewire was inserted into the duct after treating one side before treatment of the other side. In the case of multiple strictures, as many second-order branches as possible were treated. A new set of endoprostheses was inserted after completion of PDT. Additional PDT sessions were performed in a time range from 6 to 12 months thereafter.

\section{Chemotherapy}

Patients were treated with one of the following regimens: gemcitabine monotherapy, gemcitabine plus cisplatin or oxali- 
platin, gemcitabine plus capecitabine, 5-FU monotherapy, or cisplatin plus irinotecan.

\section{Statistical analysis}

The results were described using absolute numbers (percentage), mean, and standard deviation (SD). Group comparison of baseline characteristics and survival in days was done by the Mann-Whitney U-test, chi-square test, or Fisher exact test where appropriate. The Levene test showed homogeneity of variances $(p=0.38)$, therefore group comparison of survival in days was done by the Mann-Whitney U-test. The Kaplan-Meier method was used to estimate overall survival outcomes. Overall survival was calculated from the time of diagnosis to the date of death (noncensored event) or last follow-up (censored event). Twelve-month survival analysis was performed through the log-rank test if possible. Events were censored if last follow-up was within the first 365 days. Hazard ratio (HR) was calculated by univariate Cox regression analysis. A p-value of less than 0.05 were considered significant. Data were analyzed using IBM SPSS version 21.0 (IBM Corp., Armonk, NY, USA).

Table 1. Baseline Characteristics of the Hilar Cholangiocarcinoma Patients

\begin{tabular}{|c|c|c|c|c|}
\hline Characteristic & All & PDT-M & PDT-C & $\mathrm{p}$-value \\
\hline No. & 68 & 35 & 33 & \\
\hline Age at diagnosis & $64.2 \pm 10.85$ & $65.5 \pm 10.53$ & $62.8 \pm 11.17$ & $0.31^{*}$ \\
\hline Age at death & $65.4 \pm 10.98$ & $67.0 \pm 10.33$ & $64.1 \pm 11.50$ & $0.40^{*}$ \\
\hline Mean follow-up & $18.2 \pm 11.90$ & $16.3 \pm 13.30$ & $20.2 \pm 10.00$ & $0.02^{*}$ \\
\hline Female:male & 36 (52.9):32 (47.1) & 19 (54.3):16 (45.7) & 17 (51.5):16 (48.5) & $1.0^{\dagger}$ \\
\hline CC classification & & & & $0.76^{\ddagger}$ \\
\hline Bismuth type I & $1(1.5)$ & $1(2.9)$ & 0 & \\
\hline Bismuth type II & $4(5.9)$ & $2(5.7)$ & $2(6.1)$ & \\
\hline Bismuth type III & $4(5.9)$ & $2(5.7)$ & $2(6.1)$ & \\
\hline Bismuth type IV & $59(86.8)$ & $30(85.7)$ & 29 (87.9) & \\
\hline No. of PDT sessions & & & & $0.57^{\ddagger}$ \\
\hline 1 & $35(51.5)$ & $20(57.1)$ & $15(45.5)$ & \\
\hline 2 & $23(33.8)$ & $9(25.7)$ & $14(42.4)$ & \\
\hline 3 & $7(10.3)$ & $4(11.4)$ & $3(9.1)$ & \\
\hline 4 & $3(4.4)$ & $2(5.7)$ & $1(3.0)$ & \\
\hline Bilirubin at study entry & $4.9 . \pm 5.80$ & $5.8 \pm 6.70$ & $4.0 \pm 4.60$ & $0.59^{*}$ \\
\hline Positive histology/cytology & $50(73.5)$ & $24(68.6)$ & $26(78.8)$ & $0.42^{\dagger}$ \\
\hline Metastatic disease & $4(4.4)$ & $1(2.9)$ & $3(9.1)$ & $0.35^{\dagger}$ \\
\hline
\end{tabular}

Data are presented as number (\%) or mean \pm SD.

PDT-M, photodynamic monotherapy; PDT-C, photodynamic plus chemotherapy; CC, cholangiocarcinoma; PDT, photodynamic therapy.

${ }^{*}$ Mann-Whitney U-test; ${ }^{\dagger}$ Fisher exact test; ${ }^{\ddagger}$ Chi-square test.

Table 2. Chemotherapy Protocols and Reasons for Discontinuation

\begin{tabular}{|c|c|c|c|c|c|}
\hline & No. & Gemcitabine mono & Gemcitabine+cisplatin & Gemcitabine+capecitabine & Others \\
\hline \multicolumn{6}{|l|}{ Chemotherapy protocol } \\
\hline First-line & 33 & $12(36.4)$ & $7(21.2)$ & $9(27.3)$ & $5(15.2)$ \\
\hline Second-line & 9 & $6(66.7)$ & $1(11.1)$ & $1(11.1)$ & $1(11.1)$ \\
\hline Third-line & 2 & 0 & 0 & 0 & $2(100.0)$ \\
\hline \multicolumn{6}{|l|}{ Reasons for discontinuation } \\
\hline Bone marrow depression & $11(50)$ & 3 & 3 & 2 & 3 \\
\hline Refractory nausea/vomiting & $6(27.3)$ & 3 & 2 & 1 & 0 \\
\hline Infectious complications & $5(22.7)$ & 1 & 2 & 1 & 1 \\
\hline Others* & $12(54.5)$ & 3 & 6 & 2 & 1 \\
\hline
\end{tabular}

Data are presented as number (\%). Reasons for discontinuation of chemotherapy (more than one entry possible).

*e.g., drug-induced fever, flu-like syndrome, polyneuropathy, renal failure, gallbladder perforation. 


\section{RESULTS}

\section{Clinical characteristics of the patients}

Clinical data of the study population are shown in Table 1. In total, 35 patients were treated with PDT alone versus 33 patients with PDT-C. There was no significant difference regarding all clinical characteristics between the two groups such as age, gender, positive histology/cytology, Bismuth type, bilirubin at baseline, or presence of metastatic disease.

\section{Endoscopic stent placement and PDT}

All NCC patients received endoscopic stent placement after PDT. Stents were changed after 3 months on a regular basis unless clinical signs of cholangitis or stent occlusion occurred. Throughout the study, repeated endoscopic stenting was performed in both groups (PDT-M: $4.6 \pm 4.77$ stents; PDT-C: $5.42 \pm 3.47$ stents). Endoscopic stent placement of more than one stent was necessary in $64.2 \%$ and $43.6 \%$ in the groups PDT$\mathrm{M}$ and PDT-C, respectively. Percutaneous stent placement was done in similar frequencies in both groups (PDT-M: 0.89 \pm 2.35 stents; PDT-C: $0.45 \pm 0.91$ stents). None of the patients received a metal stent during the study period. Success of biliary decompression was evaluated on clinical or laboratory grounds with the help of abdominal ultrasound. There was no significant difference in bilirubin levels before (PDT-M: median, $3.5 \mathrm{mg} /$ dL, 95\% confidence interval [CI], 1.8 to 7.0; PDT-C: median, 1.9 $\mathrm{mg} / \mathrm{dL}, 95 \% \mathrm{CI}, 1.4$ to $2.5, \mathrm{p}=0.12$ ) and after PDT (PDT-M: median, $2.3 \mathrm{mg} / \mathrm{dL}, 95 \% \mathrm{CI}, 2.0$ to 5.2; PDT-C: median, $2.0 \mathrm{mg} / \mathrm{dL}$, 95\% CI, 1.3 to 3.1, $\mathrm{p}=0.11$ ). Frequency of percutaneous PDT was 5 and 6 in the groups PDT-M and PDT-C, respectively.

\section{Chemotherapy protocols}

Among NCC patients that were treated with PDT-C, the protocols are shown in Table 2. First-line chemotherapy in the majority of patients contained a gemcitabine-based regimen,

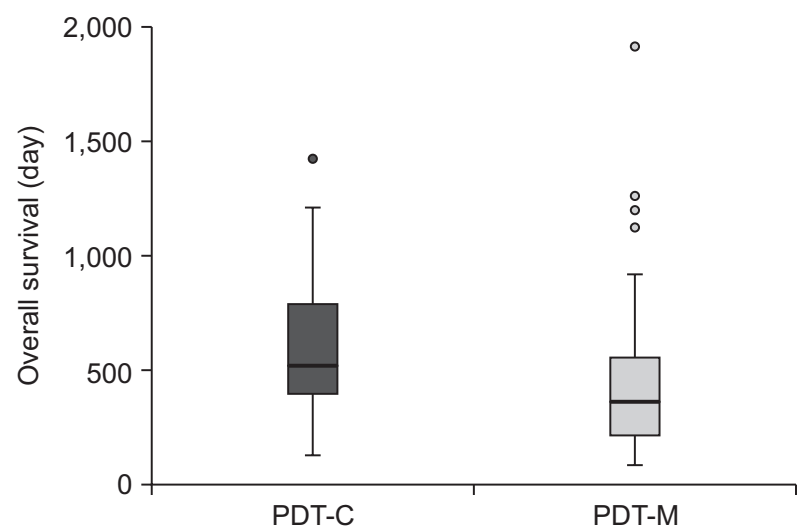

Fig. 1. Overall survival rate of the nonresectable cholangiocarcinoma patients with photodynamic monotherapy (PDT-M) and photodynamic plus chemotherapy (PDT-C) from the time of diagnosis until the time of death in days ( $p=0.021$, Mann-Whitney U-test). either as monotherapy or in combination with cisplatin or 5-FU for $(28 / 35,80.0 \%)$. The other protocols were as follows: $5-\mathrm{FU}$ monotherapy $(\mathrm{n}=1), 5-\mathrm{FU} /$ folic acid $(\mathrm{n}=2)$, cisplatin/5-FU $(\mathrm{n}=1)$, and irinotecan $(\mathrm{n}=1)$. Second-line therapy was performed in 27.3\% of patients. Among them, six patients and two patients were treated with gemcitabine monotherapy and gemcitabine combination therapy, respectively (capecitabine or cisplatin, both $n=1)$. Third-line therapy was done in two patients (capecitabine, $n=1$; gemcitabine/oxaliplatin, $n=1$ ).

The duration of chemotherapy was $9.52 \pm 8.3$ months. In total, 6 months and 12 months after inclusion, 52\% and 32\% of patients were still receiving chemotherapy, respectively. Chemotherapy was discontinued in 56\% and 100\% in patients receiving non-platinum-based therapy and platinum-based therapy, respectively. Reasons for discontinuation are shown in Table 2, the majority being cytopenia, refractory vomiting, and infectious complications.

\section{Overall survival}

Mean overall survival in all NCC patients was 483 days (95\% CI, 379 to 587). In the PDT-M group mean survival was 374 days (95\% CI, 230 to 518), whereas in the PDT-C group it was 520 days (95\% CI, 422 to 618 ; $\mathrm{p}=0.021$; Mann-Whitney Utest) (Fig. 1). This resulted in a mean survival improvement of almost 5 months in patients undergoing combination therapy. Furthermore, 1-year survival was significantly higher in the PDT-C group compared to the PDT-M group (87.9\% vs 51.4\%, $\mathrm{p}<0.001$ ) (Table 3). Kaplan-Meyer analysis within the first year showed a significant reduction for death (HR within year one, 0.20 ; $95 \%$ CI, 0.07 to $0.58 ; p=0.003$ ) (Fig. 2).

\section{Survival depending on the chemotherapy protocol}

When the different chemotherapy protocols were analyzed in the PDT-C group, the longest overall survival was found in the patients undergoing a combination therapy with gemcitabine. Herein patients with gemcitabine monotherapy had a shorter survival compared to patients undergoing combination therapy (mean, 395 days vs 566 days; $\mathrm{p}=0.09$; Mann-Whitney U-test), regardless of the other agents (cisplatin or 5-FU). There were

Table 3. Mean and Year-Dependent Survival Rates

\begin{tabular}{lcccc}
\hline \multicolumn{1}{c}{ Characteristic } & All & PDT-M & PDT-C & p-value \\
\hline No. of patients & 68 & 35 & 33 & - \\
Mean survival, day & 483 & 374 & 520 & 0.021 \\
95\% CI, day & $379-587$ & $230-518$ & $422-618$ & - \\
1-Year survival & $47(69.1)$ & $18(51.4)$ & $29(87.9)$ & $<0.001$ \\
3-Year survival & $6(8.8)$ & $4(11.4)$ & $2(6.1)$ & NS \\
5-Year survival & $1(1.5)$ & $1(2.9)$ & 0 & NS \\
\hline
\end{tabular}

Data are presented as number (\%).

PDT-M, photodynamic monotherapy; PDT-C, photodynamic plus chemotherapy; CI, confidence interval; NS, not significant. 
no significant differences between platinum-based and nonplatinum-based protocols $(\mathrm{p}=0.42)$. However, the sample size of patients in these subgroups was small.

\section{Cholangitic complications}

Cholangitic complications were observed in $76.5 \%$ of patients $(n=52)$, and $54.4 \%$ of patients $(n=37)$ had more than one cholangitic episode (Table 4). We did not observe a significant difference regarding the number of events of cholangitic complications comparing the groups PDT-M and PDT-C (Table 4). We furthermore did not find an increased risk of a specific protocol, e.g., when platinum-based versus non-platinum-based or gemcitabine monotherapy versus gemcitabine combination regimens were compared, although samples sizes in these subgroups were small (data not shown).

\section{DISCUSSION}

In this retrospective study of matched patients with hilar NCC, the combination of PDT with chemotherapy was associated with an increased 1-year survival compared to PDT alone and an overall survival improvement of almost 5 months. The risk of NCC-associated complications such as cholangitis was not increased in the chemotherapy group. Furthermore, gemcitabinebased combination chemotherapy seemed to be beneficial compared to gemcitabine monotherapy.

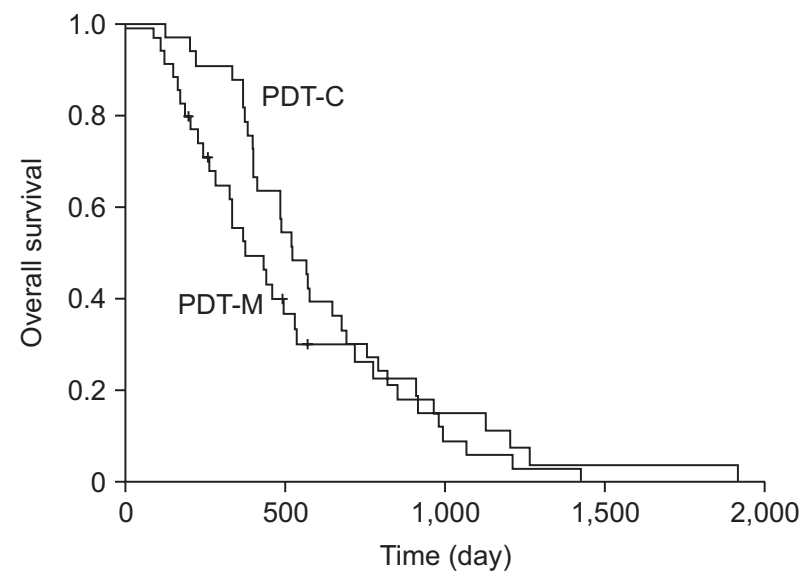

Fig. 2. Overall survival rate in photodynamic monotherapy (PDT-M) and photodynamic plus chemotherapy (PDT-C) patients.
A standard protocol for the treatment of hilar NCC is not available. Apart from best supportive care, adequate and continuous bile duct drainage via endoscopic stenting is the mainstay of palliative treatment. In addition, numerous studies have evaluated the effect of PDT on survival in NCC. Median survival ranges from 4 to more than 25 months (reviewed in Höblinger et $a l^{7}$ ), the longest survival time was observed in patients undergoing prolonged PDT (mean eight sessions). Our observation showed a mean overall survival of 16 month, which is well comparable with the results of most other studies.

In addition, various chemotherapy protocols have been analyzed and recent studies suggest an improvement in overall survival especially in gemcitabine-based combination therapies. ${ }^{6}$ Herein a recent meta-analysis showed that the best response rates and tumor control in NCC patients can be achieved by combining gemcitabine with cisplatin or oxaliplatin, ${ }^{8}$ although the differentiation between intrahepatic and hilar carcinoma is lacking in most studies.

However, data regarding combination strategies like PDT and chemotherapy are scarce. Recently a prospective study showed a significant benefit of the combination of PDT and an orally administered fluoropyrimidine, S-1 over PDT alone (17 months vs 8 months). ${ }^{9}$ A retrospective survival analysis showed that patients undergoing PDT and chemotherapy survived on average 1.8 months longer than patients receiving PDT as monotherapy. ${ }^{10}$ However, this difference was not statistically significant and the number of patients in the PDT-C group was rather small $(n=11)$. Interestingly, they also observed that patients undergoing non-gemcitabine-based chemotherapy or gemcitabinebased combination therapy had better survival than the patients receiving gemcitabine monotherapy. Another recent prospective observation showed that a similar combination therapy of PDT plus gemcitabine-based combination therapy, analyzed in 14 selected patients, resulted in 3-, 6-, and 12-month survival rates of $85 \%, 77 \%$, and 77\%, respectively. Limitations of this study are the small number of patients $(n=14)$ and the lack of a control group. ${ }^{11}$ However, this survival rate is similar to our observation (1-year survival of 88\% in the PDT-C group). Other retrospective studies included only few patients that had been treated with both PDT and chemotherapy making comparisons rather difficult. ${ }^{12,13}$

In above mentioned trial, combination therapy with gemcitabine and cisplatin resulted in the median overall survival of

Table 4. Cholangitic Complications

\begin{tabular}{llllc}
\hline \multicolumn{1}{c}{ Characteristic } & \multicolumn{1}{c}{ All } & PDT-M & PDT-C & p-value \\
\hline No. of patients & 68 & 35 & 33 & - \\
Cholangitic complications & $52(76.5)$ & $27(77.1)$ & $25(75.8)$ & 0.89 \\
$>1$ Cholangitic complications & $37(54.4)$ & $20(57.1)$ & $17(51.5)$ & 0.64 \\
\hline
\end{tabular}

Data are presented as number (\%). Cholangitic events in the nonresectable cholangiocarcinoma patients with PDT-M and PDT-C (chi-square test). PDT-M, photodynamic monotherapy; PDT-C, photodynamic plus chemotherapy. 
11.7 months analyzing all CC patients. ${ }^{6}$ In our study, the mean survival of hilar NCC patients was 17.3 months in the PDT$\mathrm{C}$ group. Baseline characteristics of both groups were similar. Furthermore, metastatic disease was even more common in the PDT-C excluding that the prolonged survival observed in these patients was due to less common systemic disease.

Our study has several limitations: first, it is a retrospective analysis and sample size is rather small. However, we focused on hilar NCC where data on the effect of PDT-C are extremely limited. Second, no standardized chemotherapy protocol was used, but this reflects the current clinical practice since no standard regimen existed in hilar NCC. Based on the recent large multicenter trial published in 2010, combination therapy of gemcitabine and cisplatin seems to be the most effective treatment in NCC. ${ }^{6}$ Since many patients were included before 2010 , only a minority of patients was treated with gemcitabine and cisplatin. There might thus be an additional benefit in overall survival if all patients would have been treated with gemcitabine plus cisplatin. Third, we did not include patients that were treated with chemotherapy and bile duct stenting alone, but PDT has shown to improve overall survival compared to best supportive care. ${ }^{3}$ Therefore we cannot exclude that PDT might have an additional benefit through improved bile duct drainage resulting in less cholangitic complications.

In summary, our study suggests that combining repeated PDT with a gemcitabine-based combination chemotherapy might offer a significant survival benefit. This is in agreement with a recently published observation from Korea, where 16 patients with PDT and chemotherapy were included. ${ }^{14}$ We also show that chemotherapy does not increase the risk for cholangitis. A combination of platinum with gemcitabine might be the preferred chemotherapy protocol adjuvant to PDT. Our data support the need for a randomized controlled trial using combination of PDT with a standardized chemotherapy in hilar NCC. We are awaiting the data of controlled and randomized trials that are currently undertaken and investigate this approach.

\section{CONFLICTS OF INTEREST}

No potential conflict of interest relevant to this article was reported.

\section{ACKNOWLEDGEMENTS}

The work is part of the doctoral thesis of Nicola Winkelmann.

\section{REFERENCES}

1. Khan SA, Thomas HC, Davidson BR, Taylor-Robinson SD. Cholangiocarcinoma. Lancet 2005;366:1303-1314.

2. Nakeeb A, Pitt HA, Sohn TA, et al. Cholangiocarcinoma: a spectrum of intrahepatic, perihilar, and distal tumors. Ann Surg 1996 224:463-473.

3. Ortner ME, Caca K, Berr F, et al. Successful photodynamic therapy for nonresectable cholangiocarcinoma: a randomized prospective study. Gastroenterology 2003;125:1355-1363.

4. Kiba T, Nishimura T, Matsumoto S, et al. Single-agent gemcitabine for biliary tract cancers: study outcomes and systematic review of the literature. Oncology 2006;70:358-365.

5. Scheithauer W. Review of gemcitabine in biliary tract carcinoma. Semin Oncol 2002;29(6 Suppl 20):40-45.

6. Valle J, Wasan H, Palmer DH, et al. Cisplatin plus gemcitabine versus gemcitabine for biliary tract cancer. N Engl J Med 2010;362: 1273-1281.

7. Höblinger A, Gerhardt T, Gonzalez-Carmona MA, Hüneburg R, Sauerbruch T, Schmitz V. Feasibility and safety of long-term photodynamic therapy (PDT) in the palliative treatment of patients with hilar cholangiocarcinoma. Eur J Med Res 2011;16:391-395.

8. Valle JW, Furuse J, Jitlal M, et al. Cisplatin and gemcitabine for advanced biliary tract cancer: a meta-analysis of two randomised trials. Ann Oncol 2014;25:391-398.

9. Park DH, Lee SS, Park SE, et al. Randomised phase II trial of photodynamic therapy plus oral fluoropyrimidine, S-1, versus photodynamic therapy alone for unresectable hilar cholangiocarcinoma. Eur J Cancer 2014;50:1259-1268.

10. Knüppel M, Kubicka S, Vogel A, et al. Combination of conservative and interventional therapy strategies for intra- and extrahepatic cholangiocellular carcinoma: a retrospective survival analysis. Gastroenterol Res Pract 2012;2012:190708.

11. Fuks D, Bartoli E, Delcenserie R, et al. Biliary drainage, photodynamic therapy and chemotherapy for unresectable cholangiocarcinoma with jaundice. J Gastroenterol Hepatol 2009;24:1745-1752.

12. Talreja JP, Degaetani M, Ellen K, Schmitt T, Gaidhane M, Kahaleh M. Photodynamic therapy in unresectable cholangiocarcinoma: not for the uncommitted. Clin Endosc 2013;46:390-394.

13. Gerhardt T, Rings D, Höblinger A, Heller J, Sauerbruch T, Schepke M. Combination of bilateral metal stenting and trans-stent photodynamic therapy for palliative treatment of hilar cholangiocarcinoma. Z Gastroenterol 2010;48:28-32.

14. Hong MJ, Cheon YK, Lee EJ, Lee TY, Shim CS. Long-term outcome of photodynamic therapy with systemic chemotherapy compared to photodynamic therapy alone in patients with advanced hilar cholangiocarcinoma. Gut Liver 2014;8:318-323. 